\title{
ON THE NOTION OF $L^{1}$-COMPLETENESS \\ OF A STOCHASTIC FLOW \\ ON A MANIFOLD
}

\author{
YU. E. GLIKLIKH AND L. A. MOROZOVA
}

Received 14 June 2002

We introduce the notion of $L^{1}$-completeness for a stochastic flow on manifold and prove a necessary and sufficient condition for a flow to be $L^{1}$-complete. $L^{1}$-completeness means that the flow is complete (i.e., exists on the given time interval) and that it belongs to some sort of $L^{1}$-functional space, natural for manifolds where no Riemannian metric is specified.

\section{Introduction}

The main goal of the paper is to investigate the conditions under which solutions of stochastic differential equations are well posed up to infinity, that is, complete. At the moment it is a well-known fact that partial differential equations of parabolic type are related to stochastic differential equations. Thus, the completeness results for stochastic differential equations are useful for parabolic equations.

Stochastic differential equations may be considered as a special generalization of ordinary differential equations, and for the latter a necessary and sufficient condition for completeness was found in [5] (see also [6]). Consider a smooth vector field (right-hand side of the equation) on a finite-dimensional manifold $M$. It was proved that all solutions are well posed for $t \in(-\infty, \infty)$ if and only if on the manifold $R \times M$ there exists a complete Riemannian metric such that the vector field $(1, X)$ is uniformly bounded with respect to it. Recall that the characteristic feature of the complete Riemannian metric is that its distance function is proper (see Definition 2.3 below).

For stochastic differential equations a certain role of the vector filed $X$, mentioned above, is played by the second order differential operator called the generator of equation. We cannot apply the technique of Riemannian metrics to second order operators but we try to replace it by Elworthy's methods from [3], where a certain sufficient condition for completeness of a stochastic differential equation in terms of values of the generator on proper functions was found. 
We introduce the notion of $L^{1}$-completeness for a stochastic flow on a manifold that is a certain modification of ordinary stochastic completeness providing the property that the flow lives in some sort of $L^{1}$-functional space, natural for manifolds where no Riemannian metric is specified. We prove that for this sort of completeness a certain condition of Elworthy's type on $\mathbb{R}_{+} \times M$ becomes necessary and sufficient. As well as for ordinary differential equation, the use of product $\mathbb{R}_{+} \times M$ is essential. As a corollary we obtain a necessary and sufficient condition of $L^{1}$-stochastic completeness for a Riemannian manifold in terms of $L^{1}$-completeness of Wiener process on the manifold.

In Section 2, we discuss our notion of $L^{1}$-completeness and give general definitions. Section 3 is devoted to the proof of the main results.

\section{Discussion and general definitions}

Let $M$ be a finite-dimensional manifold. Consider a stochastic dynamical system (SDS) on $M$ (see [3]) with the generator $\mathscr{A}(t, x)$. In local coordinates it is described in terms of a stochastic differential equation with smooth coefficients in Itô or in Stratonovich form. Since the coefficients are smooth we can pass from Stratonovich to Itô equation and vice versa. Denote by $\xi(s): M \rightarrow M, s \in[0, T]$, the stochastic flow of the SDS. For any point $x \in M$ and time $t$ the trajectory $\xi_{t, x}(s), s \in[t, T]$, of this flow is the unique solution of the above equation with initial conditions $\xi_{t, x}(t)=x$. As the coefficients of equation are smooth, this is a strong solution and a Markov diffusion process given on a certain random time interval. Below we denote the probability space, where the flow is defined by $(\Omega, \mathscr{F}, P)$ and suppose that it is complete. We also deal with separable realizations of all processes.

Definition 2.1. The flow $\xi(s)$ is complete on $[0, T]$ if $\xi_{t, x}(s)$ exists for any couple $(t, x)$ and for all $s \in[t, T]$.

Definition 2.2. The flow $\xi(s)$ is complete if it is complete on any interval $[0, T] \subset$ $\mathbb{R}$.

Let the flow $\xi(s)$ be given on the vector space $\mathbb{R}^{n}$. In this case it is natural to say that the flow $\xi(s)$ is $L^{1}$-complete on $[0, T]$ if the following assumptions hold:

(i) $\xi(s)$ is complete on $[0, T]$;

(ii) $E\left\|\xi_{t, x}(s)\right\|<\infty$ for all $t, x$, and $s \in\left[t\right.$, T] (i.e., $\xi_{t, x}(s)$ belongs to the space $L^{1}$ for any $\left.s\right)$

(iii) property (ii) holds for any $s \in[t, T]$ if it holds for at least one $s$, for example, for initial instant $t$ or final instant $T$ (i.e., if $E\left\|\xi_{t, x}(t)\right\|=\|x\|<$ $\infty$ or $\left.E\left\|\xi_{t, x}(T)\right\|<\infty\right)$;

(iv) for any $s$ the trajectory $\xi_{t, x}(s)$ is smooth enough in mean (i.e., in $L^{1}$ ) with respect to initial values $t, x$, that is $E\left\|\xi_{t, x}(s)\right\|$ is smooth enough in $t$ and in $x$. Some conditions providing such a smoothness are described, for example, in $[1,4]$. 
On an arbitrary finite-dimensional manifold $M$, in the definition of $L^{1}$ completeness the norm should be replaced by another object, satisfying the properties of norm in $\mathbb{R}^{n}$ such as its properness and smoothness.

Definition 2.3. The function $f: M \rightarrow \mathbb{R}$ is called proper if the preimage $f^{-1}(A)$ of any compact set $A \subset \mathbb{R}$ is compact in $M$.

Let $M$ be a complete Riemannian manifold. Denote by $\rho$ the distance function generated by the Riemannian metric of manifold. Specify a certain point $x_{0} \in$ $M$. For an arbitrary point $x \in M$, the distance $\rho\left(x_{0}, x\right)$ is a natural analogue of the norm in vector space. Since $M$ is complete, it follows from the Hopf-Rinow theorem (see, e.g., [2]) that the function $\rho\left(x_{0}, \cdot\right)$ is proper. Then an analogue of, for example, (ii) takes the form

$$
E \rho\left(x_{0}, \xi_{t, x}(s)\right)<\infty
$$

Unlike the norm in $\mathbb{R}^{n}$, the function $\rho$ may not be smooth at the points of cut locus. Nevertheless $\rho$ can be approximated by a smooth function with similar properties as follows.

Take $\varepsilon>0$ small enough and denote by $U_{x}(\varepsilon)$ a neighborhood of $x \in M$ that is the intersection of the metric ball with radius $\varepsilon$ (with respect to distance $\rho$ ), centered at $x$, and of some chart containing $x$. Since $M$ is paracompact, we can select a countable locally finite subcovering $\left\{U_{x_{i}}(\varepsilon)\right\}$ from the covering $\left\{U_{x}(\varepsilon)\right\}$. Let $\left\{\varphi_{i}\right\}_{i=1}^{\infty}$ be partition of unity adapted to this subcovering. Introduce the function $\rho_{\varepsilon}(x)=\sum_{i=1}^{\infty} \varphi_{i}(x) \rho\left(x_{0}, x_{i}\right)$. By the construction, function $\rho_{\varepsilon}(x)$ is smooth (unlike the distance $\rho\left(x_{0}, x\right)$ ).

Show that

$$
\left|\rho\left(x_{0}, x\right)-\rho_{\varepsilon}(x)\right|<\varepsilon,
$$

where $x$ is an arbitrary point in $M$. Indeed, from the triangle inequality we get $\left|\rho\left(x_{0}, x\right)-\rho\left(x_{0}, x_{i}\right)\right|<\rho\left(x_{i}, x\right)<\varepsilon$ for any $i$ and from the fact that the covering is locally finite it follows that

$$
\begin{aligned}
\left|\rho\left(x_{0}, x\right)-\rho_{\varepsilon}(x)\right| & =\left|\rho\left(x_{0}, x\right)-\sum_{i=p}^{m} \varphi_{i}(x) \rho\left(x_{0}, x_{i}\right)\right| \\
& \leq\left|\rho\left(x_{0}, x\right)-\max _{p \leq i \leq m} \rho\left(x_{0}, x_{i}\right) \sum_{i=p}^{m} \varphi_{i}(x)\right| \\
& =\left|\rho\left(x_{0}, x\right)-\max _{p \leq i \leq m} \rho\left(x_{0}, x_{i}\right)\right|<\varepsilon .
\end{aligned}
$$

From (2.2) one can easily see that the function $\rho_{\varepsilon}(x)$ is also proper. Moreover, from (2.2) it follows that $\rho_{\varepsilon}(x)<\rho\left(x_{0}, x\right)+\varepsilon$, so $E \rho_{\varepsilon}(\xi)<E \rho\left(x_{0}, \xi\right)+\varepsilon$ for any 
random element $\xi$ such that $E \rho\left(x_{0}, \xi\right)$ exists. Thus, if (2.1) holds,

$$
E \rho_{\varepsilon}\left(\xi_{t, x}(s)\right)<\infty
$$

So, if a complete Riemannian metric is given on a manifold $M$ and inequality (2.1) holds for the distance, there exists a smooth proper function on $M$ for which (2.4) holds. Thus, it is more natural (and more general since a metric may not be given on the manifold a priori) to introduce the notion of $L^{1}$ completeness using some proper function on $M$ with values in $\mathbb{R}$ instead of the distance so that the function has some additional properties leading to requirements analogous to (i), (ii), (iii), and (iv).

Definition 2.4. A flow $\xi(s)$ on a finite-dimensional manifold $M$ is called $L^{1}$ complete on $[0, T]$ if the following conditions are fulfilled:

(i) $\xi(s)$ is complete on $[0, T]$;

(ii) there exists a smooth proper positive function $v: M \rightarrow \mathbb{R}^{1}$ such that $E v\left(\xi_{t, x}(T)\right)<\infty$ for all $x \in M, t \in[0, T]$;

(iii) for any $K>0$ there exists a compact $C_{K, T} \subset M$, depending on $K$ and $T$, such that the inequality $E v\left(\xi_{t, x}(T)\right)<K$ yields $x \in C_{K, T}$;

(iv) the function $f(t, x)=E v\left(\xi_{t, x}(T)\right)$ is smooth.

Notice that the conditions of Definition 2.4 are weakened analogues of requirements (i), (ii), (iii), and (iv). Namely, (ii) and (iii) together mean a reduced form of assumption, analogous to (ii) and (iii) together, and smoothness, postulated in (iv), is analogous to that of (iv).

Definition 2.5. The flow $\xi(s)$ is $L^{1}$-complete if it is $L^{1}$-complete on any finite interval $[0, T] \subset \mathbb{R}_{+}=[0, \infty)$.

\section{Main statements}

Consider the direct product $M^{T}=[0, T] \times M$ and denote by $\pi^{T}: M^{T} \rightarrow M$ the natural projection: $\pi^{T}(t, x)=x$. On the manifold $M^{T}$ consider the flow $\eta(s)=$ $(s, \xi(s))$. Obviously, for any point $(t, x) \in M^{T}$ the trajectory of $\eta_{(t, x)}(s)$ satisfies the relation $\pi^{T}\left(\eta_{(t, x)}(s)\right)=\xi_{t, x}(s)$. It is clear that $\eta(s)$ is the flow generated by SDS with generator $\mathscr{A}^{T}$ determined by the formula

$$
\mathscr{A}_{(t, x)}^{T}=\mathscr{A}(t, x)+\frac{\partial}{\partial t}
$$

Let $u: M^{T} \rightarrow \mathbb{R}$ be a proper function. Consider the sequence of compacts $W_{n}=\mathcal{u}^{-1}([0, n])$ in $M^{T}$. Specify a point $(t, x) \in M^{T}$ and for $n$ such that $(t, x) \in$ $W_{n}$ denote by $\tau_{n}$ the first exit time of $\eta_{(t, x)}(s)$ from $T_{n}$.

Theorem 3.1. The flow $\xi(s)$ on $M$ is $L^{1}$-complete on $[0, T], T>0$ if and only if there exists a smooth proper positive function $u$ on $M^{T}$ such that for all $(t, x) \in M^{T}$, 
the equality $\mathscr{A}_{(t, x)}^{T} u=C$ holds where $C$ is a certain constant, and for all $(t, x) \in M^{T}$ the random variables $u\left(\eta_{(t, x)}\left(T \wedge \tau_{n}\right)\right)$ are uniformly integrable.

\section{Proof}

Sufficiency. Without loss of generality, we can suppose that $C=0$. Indeed, let $C>0$, then construct $f(t, x): M \rightarrow \mathbb{R}$, by the formula $f(t, x)=u(t, x)-C t+C T$. Evidently $f(t, x)>0$ for all $(t, x) \in M^{T}, f(t, x)$ is smooth, proper, and $\mathscr{A}_{(t, x)}^{T} f=$ 0 . If $C<0$ construct $f(t, x)=u(t, x)-C t$.

So, let there exist a smooth proper positive function $u(t, x)$ on $M^{T}$ such that $\mathscr{A}_{(t, x)}^{T} u=0$ at all points of $M^{T}$. Then from [3, Theorem IX. 6A] it follows that $\eta(s)$ is complete, that is, the trajectories $\eta_{(t, x)}(s)=\left(s, \xi_{t, x}(s)\right)$ do exist for all points $(t, x) \in M^{T}$ and for all $s \in[t, T]$. Obviously this means that the flow $\xi(s)$ is also complete.

Consider the sequence of compacts $W_{n}=u^{-1}([0, n])$ in $M^{T}$ and the corresponding sequence of first exit times $\tau_{n}$ introduced above.

Taking into account Itô formula and the definition of generator and applying the machinery of [3] (see Corollary III.7C and the proof of Theorem IX. 6A there) one easily obtains that

$$
E u\left(\eta_{(t, x)}\left(T \wedge \tau_{n}\right)\right)=u(t, x)+E \frac{1}{2} \int_{t}^{T \wedge \tau_{n}} \mathscr{A}_{\eta_{(t, x)}(s)}^{T} u d s .
$$

Since $\mathscr{A}_{\eta_{(t, x)}(s)}^{T} u=0$ by the hypothesis, we have

$$
E u\left(\eta_{(t, x)}\left(T \wedge \tau_{n}\right)\right)=u(t, x) .
$$

Immediately from the construction, it follows that the random variables $u\left(\eta_{(t, x)}\left(T \wedge \tau_{n}\right)\right)$ a.s. converge to $u\left(\eta_{(t, x)}(T)\right)$. Since by the hypothesis the variables $u\left(\eta_{(t, x)}\left(T \wedge \tau_{n}\right)\right)$ are uniformly integrable, this means that

$$
E u\left(\eta_{(t, x)}(T)\right)=u(t, x) .
$$

Consider the function

$$
v(x)=u(T, x) .
$$

From (3.4) and (3.5) it follows that

$$
E v\left(\xi_{t, x}(T)\right)=E u\left(T, \xi_{t, x}(T)\right)=E u\left(\eta_{(t, x)}(T)\right)=u(t, x) .
$$

Thus, $E v\left(\xi_{t, x}(T)\right)<\infty$ and $E v\left(\xi_{t, x}(T)\right)$ is smooth as a function of variables $(t, x)$.

Suppose that $E v\left(\xi_{t, x}(T)\right)<K$, then $E u\left(\eta_{(t, x)}(T)\right)=u(t, x)<K$. Since $u$ is positive, this means that the value $u(t, x)$ belongs to the compact $[0, K] \subset \mathbb{R}$ and since $u$ is proper, the set $u^{-1}([0, K]) \subset M^{T}$ is compact. Thus if $E v\left(\xi_{t, x}(T)\right)<K$, the point $x$ belongs to the compact $\pi^{T}\left(u^{-1}([0, K])\right)$. 
So, conditions (i), (ii), (iii), and (iv) of Definition 2.4 are fulfilled and the flow $\xi(s)$ is $L^{1}$-complete. This proves the sufficiency.

Necessity. Let the flow $\xi(s)$ on $M$ be $L^{1}$-complete on $[0, T]$. Denote by $v: M \rightarrow \mathbb{R}$ the smooth proper positive function from Definition 2.4. Consider the function $u(t, x)=E v\left(\xi_{t, x}(T)\right)$. By (iv) of Definition 2.4 it is smooth.

Since $v$ is proper, the sets $\tilde{W}_{n}=v^{-1}([0, n])$ are compact. Denote by $\tilde{\tau}_{n}$ the first exit time of $\xi_{t, x}(s)$ from $\tilde{W}_{n}$. Consider

$$
E u\left((t+\Delta t) \wedge \tilde{\tau}_{n}, \xi_{t, x}\left((t+\Delta t) \wedge \tilde{\tau}_{n}\right)\right)=E u\left(\eta_{(t, x)}\left((t+\Delta t) \wedge \tilde{\tau}_{n}\right)\right)
$$

Since the sample trajectories of $\xi_{(t, x)}$ are considered up to the first exit time from compact $\tilde{W}_{n}$, we can apply Itô formula. Hence, since the expectation of Itô integral here is equal to zero,

$$
E u\left((t+\Delta t) \wedge \tilde{\tau}_{n}, \xi_{t, x}\left((t+\Delta t) \wedge \tilde{\tau}_{n}\right)\right)=u(t, x)+E \int_{t}^{(t+\Delta t) \wedge \tilde{\tau}_{n}} \mathscr{A}^{T} u(\eta(t, x)(s)) d s
$$

Notice that $E u\left((t+\Delta t) \wedge \tilde{\tau}_{n}, \xi_{t, x}\left((t+\Delta t) \wedge \tilde{\tau}_{n}\right)\right)=u(t, x)$. Indeed,

$$
\begin{aligned}
E u\left((t+\Delta t) \wedge \tilde{\tau}_{n}, \xi_{t, x}\left((t+\Delta t) \wedge \tilde{\tau}_{n}\right)\right) & =E\left(E v\left(\xi_{(t+\Delta t) \wedge \tilde{\tau}_{n}, \xi_{t, x}\left((t+\Delta t) \wedge \tilde{\tau}_{n}\right)}(T)\right)\right) \\
& =E\left(E v\left(\xi_{t, x}(T)\right)\right)=E v\left(\xi_{t, x}(T)\right) \\
& =u(t, x)
\end{aligned}
$$

Then, taking into account (3.8), we obtain

$$
\begin{aligned}
0 & =E u\left((t+\Delta t) \wedge \tilde{\tau}_{n}, \xi_{t, x}\left((t+\Delta t) \wedge \tilde{\tau}_{n}\right)\right)-u(t, x) \\
& =E \int_{t}^{(t+\Delta t) \wedge \tilde{\tau}_{n}} \mathscr{A}^{T} u(\eta(t, x)(s)) d s .
\end{aligned}
$$

Multiply the equality (3.10) by $1 / \Delta t$ and pass to the limit as $\Delta t \rightarrow 0$. Then, taking into account (3.1), we get

$$
\begin{aligned}
0 & =\lim _{\Delta t \rightarrow 0} \frac{1}{\Delta t} E \int_{t}^{(t+\Delta t) \wedge \tilde{\tau}_{n}} \mathscr{A}^{T} u(\eta(t, x) \\
& =\lim _{\Delta t \rightarrow 0} \frac{1}{\Delta t} E \int_{t}^{(t+\Delta t) \wedge \tilde{\tau}_{n}}\left(\frac{\partial u\left(s, \xi_{t, x}(s)\right)}{\partial s}+\mathscr{A} u\left(s, \xi_{t, x}(s)\right)\right) d s
\end{aligned}
$$

Recall again that in the case under consideration the process $\xi_{t, x}(s)$ belongs to the compact $\tilde{W}_{n}$, hence the function $u$ is considered on compact $[0, T] \times \tilde{W}_{n}$ and so its values are bounded. This means that we can apply Lebesgue's theorem on passing to limit under integral (i.e., expectation) and that there exists a random 
variable $s^{\prime} \in\left[t,(t+\Delta t) \wedge \tilde{\tau}_{n}\right]$ such that

$$
\begin{aligned}
\int_{t}^{(t+\Delta t) \wedge \tilde{\tau}_{n}}\left(\frac{\partial u\left(s, \xi_{t, x}(s)\right)}{\partial s}+\mathscr{A} u\left(s, \xi_{t, x}(s)\right)\right) d s \\
=\left[\frac{\partial u\left(s^{\prime}, \xi_{t, x}\left(s^{\prime}\right)\right)}{\partial s}+\mathscr{A} u\left(s^{\prime}, \xi_{t, x}\left(s^{\prime}\right)\right)\right]\left((t+\Delta t) \wedge \tilde{\tau}_{n}-t\right) .
\end{aligned}
$$

One can easily see that $(t+\Delta t) \wedge \tilde{\tau}_{n}-t=((t+\Delta t)-t) \wedge\left(\tilde{\tau}_{n}-t\right)=\Delta t \wedge\left(\tilde{\tau}_{n}-t\right)$. Thus

$$
\begin{aligned}
0 & =\lim _{\Delta t \rightarrow 0} \frac{1}{\Delta t} E\left(\frac{\partial u\left(s^{\prime}, \xi_{t, x}\left(s^{\prime}\right)\right)}{\partial s}+\mathscr{A} u\left(s^{\prime}, \xi_{t, x}\left(s^{\prime}\right)\right)\right)\left(\Delta t \wedge\left(\tilde{\tau}_{n}-t\right)\right) \\
& =E \lim _{\Delta t \rightarrow 0}\left(\frac{\partial u\left(s^{\prime}, \xi_{t, x}\left(s^{\prime}\right)\right)}{\partial s}+\mathscr{A} u\left(s^{\prime}, \xi_{t, x}\left(s^{\prime}\right)\right)\right) \frac{\Delta t \wedge\left(\tilde{\tau}_{n}-t\right)}{\Delta t} .
\end{aligned}
$$

Notice that $\tilde{\tau}_{n}-t>0$ a.s., since at $t$ the process $\xi_{t, x}$ takes the value $x$ and so $t$ cannot be the first exit time from $\tilde{W}_{n}$, and that $\tilde{\tau}_{n}-t$ is bounded and does not depend on $\Delta t$. Hence

$$
\lim _{\Delta t \rightarrow 0} \frac{\tilde{\tau}_{n}-t}{\Delta t}=\infty
$$

and so

$$
\lim _{\Delta t \rightarrow 0} \frac{\Delta t \wedge\left(\tilde{\tau}_{n}-t\right)}{\Delta t}=1 \wedge \lim _{\Delta t \rightarrow \infty} \frac{\tilde{\tau}_{n}-t}{\Delta t}=1 .
$$

From $s^{\prime} \in\left[t,(t+\Delta t) \wedge \tilde{\tau}_{n}\right]$ and from Lebesgue's theorem it follows that $s^{\prime} \rightarrow t$ as $\Delta t \rightarrow 0$. So, equality (3.13) takes the form

$$
\frac{\partial u\left(t, \xi_{t, x}(t)\right)}{\partial t}+\mathscr{A} u\left(t, \xi_{t, x}(t)\right)=0
$$

This means that

$$
\mathscr{A}^{T} u(t, x)=0 .
$$

Lemma 3.2. The function $u$ on $M^{T}$ is proper.

Proof. Suppose that $u$ is not proper. Then there exists a sequence of points $\left(t_{k}, x_{k}\right)$ $\in M^{T}$ such that $0<u\left(t_{k}, x_{k}\right)<K$ for all $k$, where $K>0$ is a certain real number, while $v\left(x_{k}\right) \rightarrow \infty$ as $k \rightarrow \infty$. Since $v$ is proper this means that $x_{k}$ leaves any specified compact in $M$.

However, if $0<u\left(t_{k}, x_{k}\right)<K$, then $E v\left(\xi_{t_{k}, x_{k}}(T)\right)<K$ and since the flow $\xi(s)$ is $L^{1}$-complete, it follows from (iii) of Definition 2.4 that $x_{k}$ belong to a certain compact $C_{K, T}$.

Introduce compact sets $W_{n}=u^{-1}([0, n])$ and denote by $\tau_{n}$ the first exit time of $\eta_{(t, x)}(s)$ from $W_{n}$. Consider the random variables $u\left(\eta_{(t, x)}\left(T \wedge \tau_{n}\right)\right)$. Taking into 
account the Itô formula, the properties of mathematical expectation and the equality $\mathscr{A}^{T} u(t, x)=0$ proved above, we obtain

$$
E u\left(\eta_{(t, x)}\left(T \wedge \tau_{n}\right)\right)=u(t, x)
$$

for each $n$.

From the construction of function $u$ and flow $\eta$ we also get

$$
E u\left(\eta_{(t, x)}(T)\right)=E v\left(\xi_{t, x}(T)\right)=u(t, x)
$$

As well as in the proof of sufficiency one can easily see that the sequence $u\left(\eta_{(t, x)}\left(T \wedge \tau_{n}\right)\right)$ tends a.s. to $u\left(\eta_{(t, x)}(T)\right)$. Thus, from (3.18) and (3.19) it follows that

$$
E \lim _{n \rightarrow \infty} u\left(\eta_{(t, x)}\left(T \wedge \tau_{n}\right)\right)=E u\left(\eta_{(t, x)}(T)\right)=u(t, x)
$$

and this means that the random variables $u\left(\eta_{(t, x)}\left(T \wedge \tau_{n}\right)\right)$ are uniformly integrable. This completes the proof of necessity. The theorem follows.

Corollary 3.3. The flow $\xi(s)$ on $M$ is $L^{1}$-complete if and only if for any finite interval $[0, T]$ there exists a smooth proper positive function $u_{T}$ on $M^{T}$ such that for any $(t, x) \in M^{T}$, the equality $\mathscr{A}_{(t, x)}^{T} u_{T}=C^{T}$ holds, where $C^{T}$ is a certain constant that can depend on $T$ and for each $T$ the random variables $u_{T}\left(\eta_{(t, x)}\left(T \wedge \tau_{n}\right)\right)$ are uniformaly integrable.

Recall the following definition.

Definition 3.4. A Riemannian manifold $M$ is called stochastically complete if the flow of SDS with generator $(1 / 2) \Delta$ (Laplace-Beltrami operator) is complete.

Denote the above-mentioned flow by $w(s)$. For any $x \in M$, the corresponding $w_{0, x}(s)$ is a Wiener process on $M$ starting at $x$.

Definition 3.5. A Riemannian manifold $M$ is called $L^{1}$-stochastically complete if the flow $w(s)$ is $L^{1}$-complete.

Introduce the operator $\Delta^{T}=\Delta+\partial / \partial t$ on $M^{T}=[0, T] \times M$.

THeorem 3.6. A Riemannian manifold $M$ is $L^{1}$-stochastically complete if and only if for any $T>0$ on the manifold $M^{T}=[0, T] \times M$, there exists a smooth proper positive function $u_{T}$ such that for all $(t, x) \in M^{T}$, the values $\Delta_{(t, x)}^{T} u_{T}$ are equal to a certain constant that may depend on $T$, and such that the random variables $u(T \wedge$ $\left.\tau_{n}, w_{t, x}\left(T \wedge \tau_{n}\right)\right)$ are uniformly integrable.

Theorem 3.6 follows from Corollary 3.3. 


\section{Acknowledgment}

This work was partially supported by grant 99-00559 from INTAS, by grant UR.04.01.008 of the Program "Universities of Russia" and by U.S. CRDF-RF Ministry of Education Award VZ-010-0. The authors are indebted to K. D. Elworthy for very useful discussions.

\section{References}

[1] Ya. I. Belopol'skaya and Yu. L. Dalecky, Stochastic Equations and Differential Geometry, Mathematics and Its Applications (Soviet Series), vol. 30, Kluwer Academic Publishers, Dordrecht, 1990.

[2] R. L. Bishop and R. J. Crittenden, Geometry of Manifolds, Pure and Applied Mathematics, vol. 15, Academic Press, New York, 1964.

[3] K. D. Elworthy, Stochastic Differential Equations on Manifolds, London Mathematical Society Lecture Note Series, vol. 70, Cambridge University Press, Cambridge, 1982.

[4] I. I. Gihman and A. V. Skorokhod, Introduction to the Theory of Random Processes, 2nd ed., Izdat. Nauka, Moscow, 1977 (Russian).

[5] Ju. E. Gliklih, Conditions for nonlocal continuability of the integral curves of vector fields, Differencial'nye Uravnenija 13 (1977), no. 4, 743-744 (Russian).

[6] Yu. Gliklikh, Global Analysis in Mathematical Physics. Geometric and Stochastic Methods, Applied Mathematical Sciences, vol. 122, Springer-Verlag, New York, 1997.

Yu. E. Gliklikh: Mathematics Faculty, Voronezh State University, 394006 Voronezh, Russia

E-mail address: yeg2000@pisem.net, yuri@yeg.vsu.ru

L. A. Morozova: Mathematics Faculty, Voronezh State University, 394006 VORONEZH, RUSSIA 


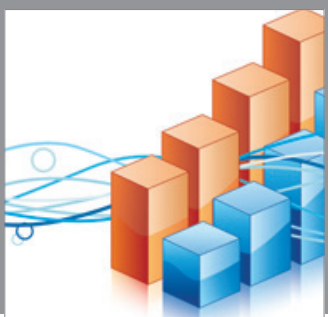

Advances in

Operations Research

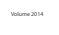

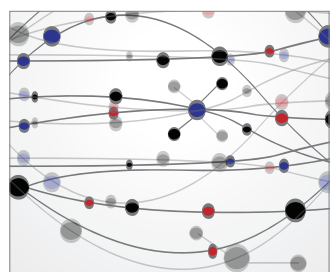

\section{The Scientific} World Journal
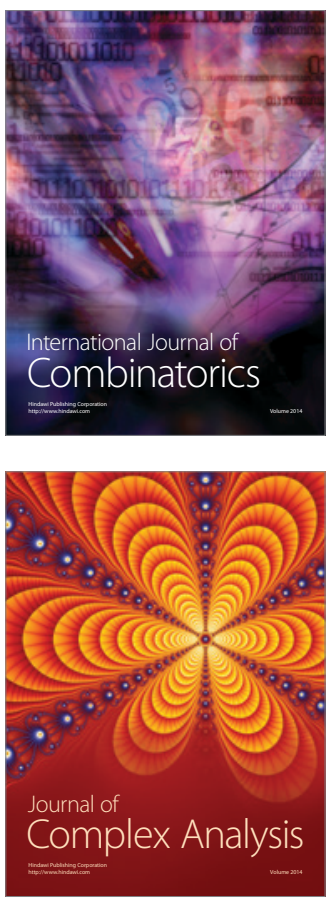

International Journal of

Mathematics and

Mathematical

Sciences
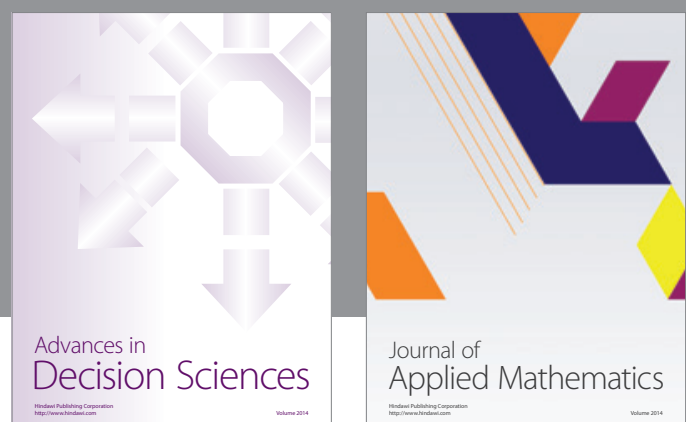

Journal of

Applied Mathematics
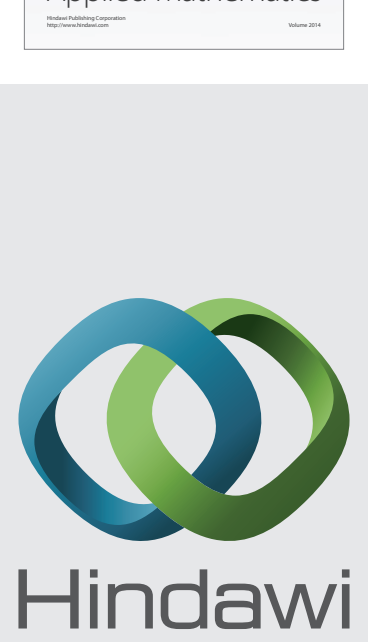

Submit your manuscripts at http://www.hindawi.com
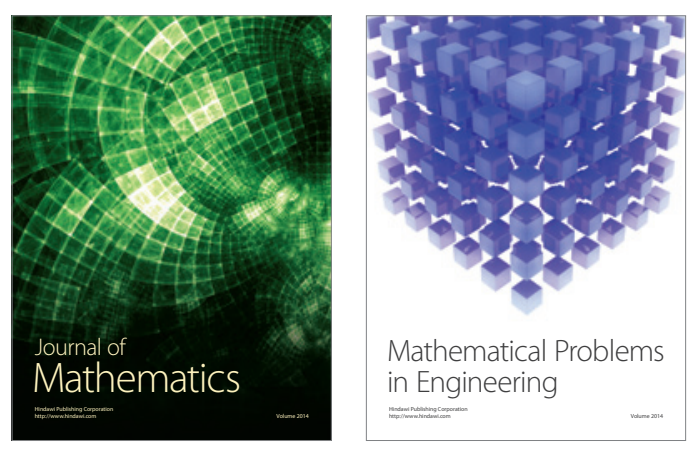

Mathematical Problems in Engineering
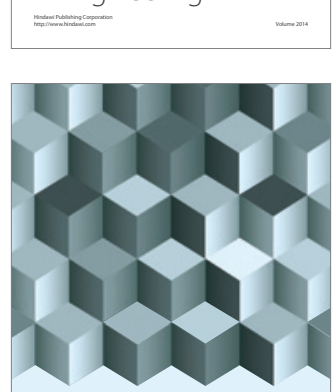

Journal of

Function Spaces
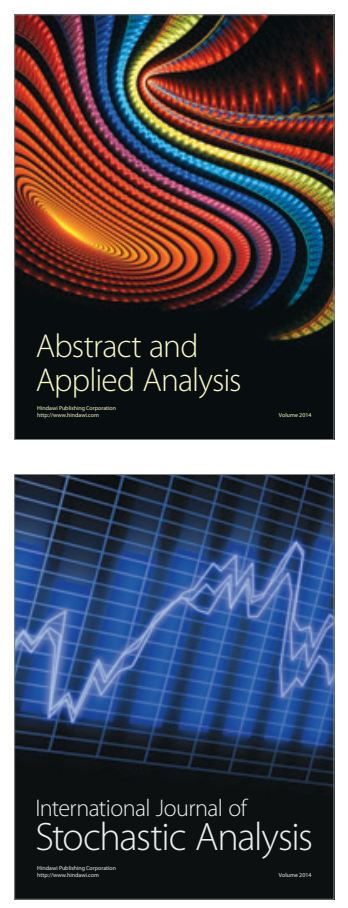

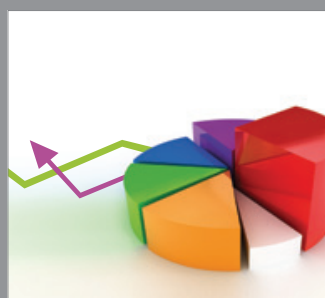

ournal of

Probability and Statistics

Promensencen
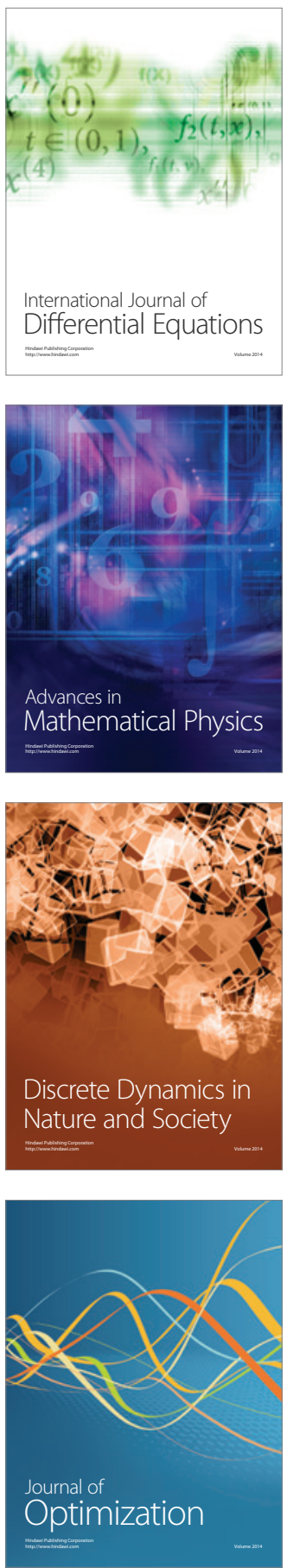